Article

\title{
Analysis of Ionicity-Magnetism Competition in 2D-MX3 Halides towards a Low-Dimensional Materials Study Based on GPU-Enabled Computational Systems
}

\author{
Alexey Kartsev ${ }^{1, *(\mathbb{D})}$, Sergey Malkovsky ${ }^{1}\left[\right.$ and Andrey Chibisov ${ }^{1,2} \mathbb{( 1 )}$ \\ 1 Computing Center of the Far Eastern Branch of the Russian Academy of Sciences, 680000 Khabarovsk, Russia; \\ sergey.malkovsky@ccfebras.ru (S.M.); andreichibisov@yandex.ru (A.C.) \\ 2 Pacific National University, 680035 Khabarovsk, Russia \\ * Correspondence: karec1@gmail.com
}

Citation: Kartsev, A.; Malkovsky, S.; Chibisov, A. Analysis of Ionicity-Magnetism Competition in 2D-MX3 Halides towards a

Low-Dimensional Materials Study Based on GPU-Enabled

Computational Systems.

Nanomaterials 2021, 11, 2967. https://

doi.org/10.3390/nano11112967

Academic Editor: Seung Geol Lee

Received: 18 August 2021

Accepted: 22 October 2021

Published: 5 November 2021

Publisher's Note: MDPI stays neutral with regard to jurisdictional claims in published maps and institutional affiliations.

\begin{abstract}
The acceleration of parallel high-throughput first-principle calculations in the context of 3D (three dimensional) periodic boundary conditions for low-dimensional systems, and particularly 2D materials, is an important issue for new material design. Where the scalability rapidly deflated due to the use of large void unit cells along with a significant number of atoms, which should mimic layered structures in the vacuum space. In this report, we explored the scalability and performance of the Quantum ESPRESSO package in the hybrid central processing unit - graphics processing unit (CPU-GPU) environment. The study carried out in the comparison to CPU-based systems for simulations of 2D magnets where significant improvement of computational speed was achieved based on the IBM ESSL SMP CUDA library. As an example of physics-related results, we have computed and discussed the ionicity-covalency and related ferro- (FM) and antiferro-magnetic (AFM) exchange competitions computed for some $\mathrm{CrX}_{3}$ compounds. Further, it has been demonstrated how this exchange interplay leads to high-order effects for the magnetism of the $1 \mathrm{~L}-\mathrm{RuCl}_{3}$ compound.
\end{abstract}

Keywords: density functional theory; 2D magnets; $\mathrm{CrI} 3$; $\mathrm{RuCl} 3$; biquadratic exchange; ferromagentism; CUDA; ESSL; CPU; GPU

\section{Introduction}

Since the first discovery of graphene, 2D materials have been proposed as a designing basis of novel quantum systems and devices for applications in energy harvesting and microelectronics. Recently, for the first time, the monolayer $2 \mathrm{D} \mathrm{CrI}_{3}$, ferromagnet with a honeycomb Cr-sublattice has been fabricated [1], followed by the cascade of experimental and theoretical studies about a new class of 2D magnets [2]. In that fashion high-throughput simulation of 2D magnets is now an emerging field in solid-state physics [2-4], with a growing need for new parallel computational methods based on various numerical techniques. First-principal understanding and predicting of 2D magnetic system properties from the computational point of view is challenging because of the need to capture the whole complexity of the interplay between quantum effects. Where large-scale nanoconstructions, such as $\mathrm{CrI}_{3}$ nanotubes, show even more fascinating electronic and magnetic properties [5]. Therein marks the need for efficiency improvement of first-principle codes and their scaling over on high-performance systems.

In the last decade GPUs have been actively developing the direction of high-performance computing systems, where classical CPU-based systems reached their limits. They have shown high computational efficiency and, therefore, have been increasingly used for a simulation purpose in the various fields of physics [6-8], where an optimal choice for configuration of the parallel environment is the key point to reach the best performance [9]. In this work, we focus on the testing and comparison of computer design methods applied to these materials in the frame of the Quantum ESPRESSO package using both CPU and 
GPU enhanced versions. The main branch of GPU-accelerated Quantum ESPRESSO- pw.x module has been tested for electronic properties calculations. Computational costs and scaling tests have been performed using the hybrid GPU-CPU environment and the pure CPU environment where the $2 \mathrm{D} \mathrm{CrI}_{3}$ magnets have been used as a testing system. Two types of pseudopotentials have been employed - using the ultrasoft pseudopotentials (US) approach and the projector augmented-wave method (PAW).

\section{Hardware and Parallel Environment}

The hybrid computing cluster used consists of one control and four computing nodes. They are Sitonica PW22LC servers (IBM Power System S822LC 8335-GTB). Where each node includes two IBM POWER8 processors with a maximum frequency of $4.023 \mathrm{GHz}$, two NVIDIA Tesla P100 GPU co-processors (NVIDIA Corporation, 2701 San Tomas Expressway, Santa Clara, CA, USA), 256 GB DDR4 RAM, an EDR InfiniBand controller, and two Seagate ST1000NX0313 1 TB 7200RPM hard drives (Seagate Technology, Inc. 10200 South De Anza Boulevard, Building One, Cupertino, CA, USA). In order to store data, a storage area network with $50 \mathrm{~TB}$ capacity was used. A management network based on Gigabit Ethernet technology was used. While for the data network, the EDR InfiniBand network with a capacity of $100 \mathrm{~Gb} / \mathrm{s}$ was employed.

For comparison purposes, three different builds of Quantum ESPRESSO (QE) version 6.2 open-source package were used. The first one (CPU) is a simple CPU-version without GPU-support, in which as a mathematical library, the serial version of the IBM ESSL version 6.2.1 (Engineering and Scientific Subroutine Library) library was used. The second one (GPU) is the Quantum ESPRESSO version 6.2 with GPU-support activated with a special open-source extension QE-GPU-Plugin [10].

The serial version of the IBM ESSL version 6.2.1 library was also used as a mathematical library in this build. The third one (ESSLCUDA) is the standard CPU-version compiled along with the GPU-enabled version of the ESSL library, which supports the offloading part of the calculations into co-processors. All versions were compiled by IBM compilers (IBM XL Fortran version 15.1.5, IBM XL C \C++ version 13.1.5) with the activated support of the parallel execution in the frame of IBM spectrum Message Passing Interface (MPI) version 10.1.0.

Calculations have been performed with the use of 1-3 computational nodes (8-60 cores, 1-6 co-processors respectively) where each MPI-process corresponds to one processor core. Their binding to co-processors was performed by the setup of the CUDA_VISIBLE_DEVICES environment variable so that MPI processes running on the first processor cores would be linked to the first co-processor, and processes of the second processor cores would be linked to the second co-processor. Calculations were terminated after performing the full self-consistent loop for each case.

The CPU machine has been used for the comparison is the Cray XC30 (Cray Inc. 901 Fifth Avenue, Suite 1000 Seattle, WA, USA) where each compute-node contains two Intel 2.7 GHz 12-core E5-2697 v2 (Ivy Bridge) series processors with the supported two hardware threads per core. The two processors are connected by two QuickPath Interconnect (Intel Corporation 2200 Mission College Blvd. Santa Clara, CA, USA) links with local memory of $32 \mathrm{~GB}$. For the sake of consistency in the second $\mathrm{CrI}_{3}$ case, the same Quantum ESPRESSO 6.2 version was used coherently both on the Cray and POWER8 machines, while the first system proceeded using version 5.4 .

\section{Computational Details}

The ground states calculations were performed using a computational implementation of the density-functional theory (DFT) package Quantum ESPRESSO [11]. We used an ultrasoft (US) pseudopotential approach along with the PAW method and the PerdewBurke-Ernzerhof generalized gradient approximation [12] of the exchange and correlation functional within its spin-polarized version. For scalar relativistic and relativistic pseudopotentials, the 600 Ryd charge density cut-off and $60 \mathrm{eV}$ wave function cut-off was 
employed to optimize the ground state. The $18 \times 18 \times 1$ (for a double layer material) and $7 \times 7 \times 1$ (for the single layer material) Monkhorst and Pack $k$-point meshes [13] were used for integration in the irreducible Brillouin zone by a special-points technique with broadening $\sigma=0.02$ Ry according to the Marzari-Vanderbilt cold smearing method [14]. Thus, these meshes ensure convergence of total energy to less than $10^{-6} \mathrm{eV} /$ atom. By processing the output data from Quantum ESPRESSO, the Hamilton-weighted populations were calculated based on periodic plane-wave DFT output with the aid of the 'LOBSTER' software package [15]. For the treatment of multi-layered systems, Van der Waals corrections were used based on the semiempirical Grimme's DFT-D2 approach.

It is well-known that for $d$-electrons in chromium compounds one has to take into account correlation effects in order to describe their electronic and magnetic properties correctly [16,17]. A density functional theory plus Hubbard U method is the most efficient and widely used approach to investigate strongly correlated semiconductors [18]. In this paper, for the main GGA+U calculations in the frame of DFT, we employed the simplified scheme introduced by Dudarev et al. [19]. We used $U_{e} f f$ values which have been computed previously using a linear response method [20]-for $2 \mathrm{D} \mathrm{CrI}_{3}, \mathrm{CrBr}_{3}$ and $\mathrm{CrCl}_{3} U_{e} f f$ are equal to $2.65 \mathrm{eV}, 2.68 \mathrm{eV}$, and $2.63 \mathrm{eV}$, respectively. For the treatment of $5 d / 4 d$ electron correlation effects in the Ru-based compounds, we used the recommended value $1.6 \mathrm{eV}$ [21] for $U_{e} f f$.

\section{Computational Performance}

In the first instance, we performed a standard QE PSIWAT test [11], which allowed us to identify the best-performed build version of the code. The corresponding test system is a gold surface covered by thiols in interaction with water (see Figure 1). Three versions of the software builds have been tested-CPU, GPU, and ESSLCUDA. Benchmarks result for the computational acceleration $S$ as a function of processors number $N$ are presented on the Figure. Where the three abovementioned builds of the code were employed. The acceleration has been computed as follow $S=T_{8} / T_{N}$, where $T_{8}$ is the average wall time using 8 cores for the first build version of the code, and $T_{N}$-is an average wall time of code in the consideration. Looking onto Figure 1, it is clear that for any number of MPI processors, GPU and ESSLCUDA builds pose the best performance over the co-processor disable version. Furthermore, with the use of the GPU-enabled version, it was possible to performer calculations about 3.3 times faster, where, in addition to eight cores, one coprocessor was involved. The best performance was observed for the ESSLCUDA build of the package. The minimal wall time reached is 553 seconds, which is 2.1 and 1.6 times faster than testing results for the CPU and GPU build on the same cluster. In the interest of understanding the influence of k-point parallelization onto the performance, we also performed calculations with the use of only one k-points pool (npool = 1). This clearly indicates the performance degradation against the k-grid distribution over 4-nodes (npool = 4), where computational enhancements can be achieved by increasing the number of MPI-processors in use.

The next step was with the use of the best performed QE build to compare its performance with other nation-wide top-ranking supercomputer facilities. The Cray XC30 MPP supercomputer equipped with a high-performance Lustre storage system was chosen as its counterpart. To emphasize the role of the unit cell size and k-points mesh for a purer 2D system, there are two test cases that have been considered.

Case 1 is the mono-layered system containing $4 \mathrm{Cr}$ atoms and $12 \mathrm{I}$ atoms with a relatively dense k-points mesh $18 \times 18 \times 1$ (164 k-points in total) and extremely large vacuum space $43 \AA$ where PAW pseudopotentials have been used. The second case is a single layer of $\mathrm{CrI}_{3}$ (25 k-points in total) where smaller vacuum space $25 \AA$ and loose $7 \times 7 \times 1$ k-points mesh have been applied along with US pseudopotentials. The aim here was to make the system computationally heavier not only by including more atoms but also by utilizing a more dense grid. This should reveal how efficient the k-points parallelization implemented into the QE package is. 
The results of the scaling analysis are presented in Figure 2. This graph is quite revealing in several ways. First, unlike the reasonable expectations, it is clear that the scalability for Case 2 reaches the limitation. Case 2 scaling was better and related to the presence of a relativity larger number of k-points, where each k-point pool can process separately on each computational core. Secondly, performing polynomial fitting of the results, we identified that a quadratic term $\left(\propto 1 / N^{2}\right)$ significantly contributed to $T(N)$ trends. However, this can be explained as a processing slowing due to the inter-node connection where $\mathrm{N} \times \mathrm{N}$ operations need to be performed. Furthermore, it can be resolved by using the -npool option for k-points distribution over separate nodes. At the same time, what can be clearly seen in this figure is the constant dominance of the hybrid system performance. Obtained results for a double-layered system of $\mathrm{CrI}_{3}$ magnet presented on Figure 3.
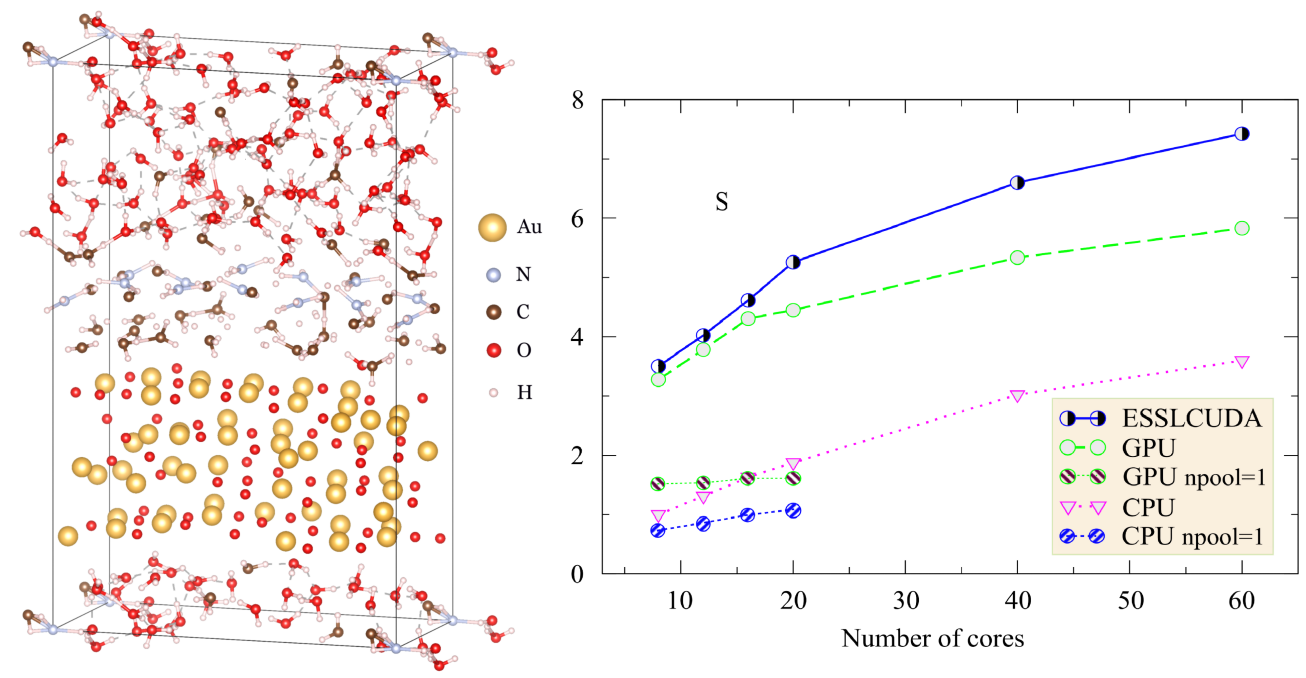

Figure 1. The orthorhombic unit cell used for simulating moistening of the gold surface coated with thiol. The second panel shows computational acceleration as a function of core number for different assemblies used for that cases calculations.

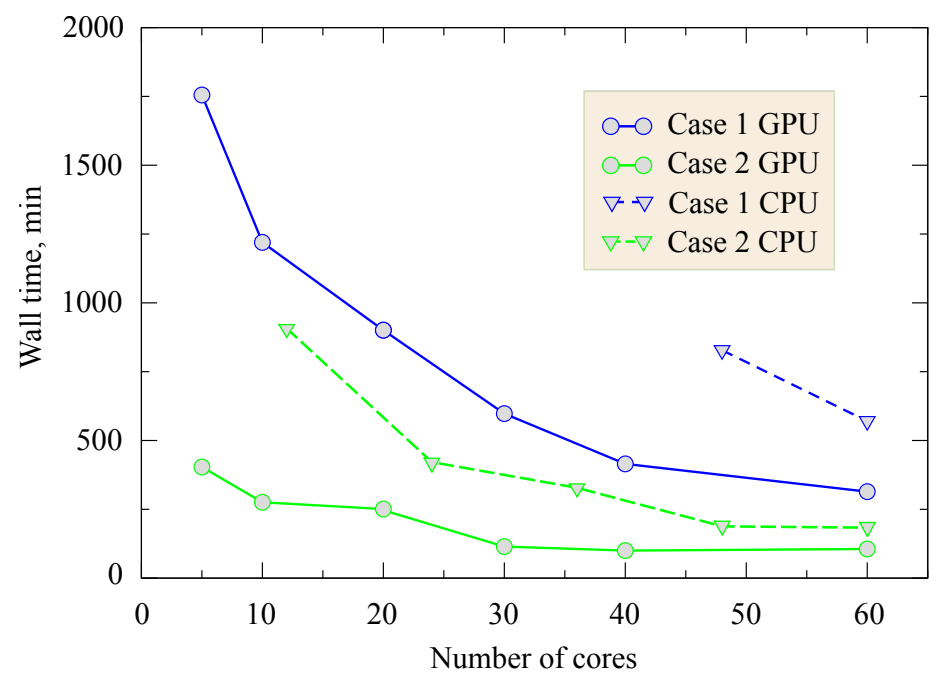

Figure 2. Wall time as a function of core number for different cases used for test calculations. Computational acceleration as a function of MPI core number. Case 1 (hard case) is the monolayer (4 Cr and 12 I atoms) k-points mesh $18 \times 18 \times 1$ (164 k-points in total) and vacuum space $43 \AA$. Case 2 (mild case) is the single layer of $\mathrm{CrI}_{3}$ (25 k-points in total) where smaller vacuum space $25 \AA$ and loosened $7 \times 7 \times 1$ k-points mesh have been applied along with US pseudopotentials. 


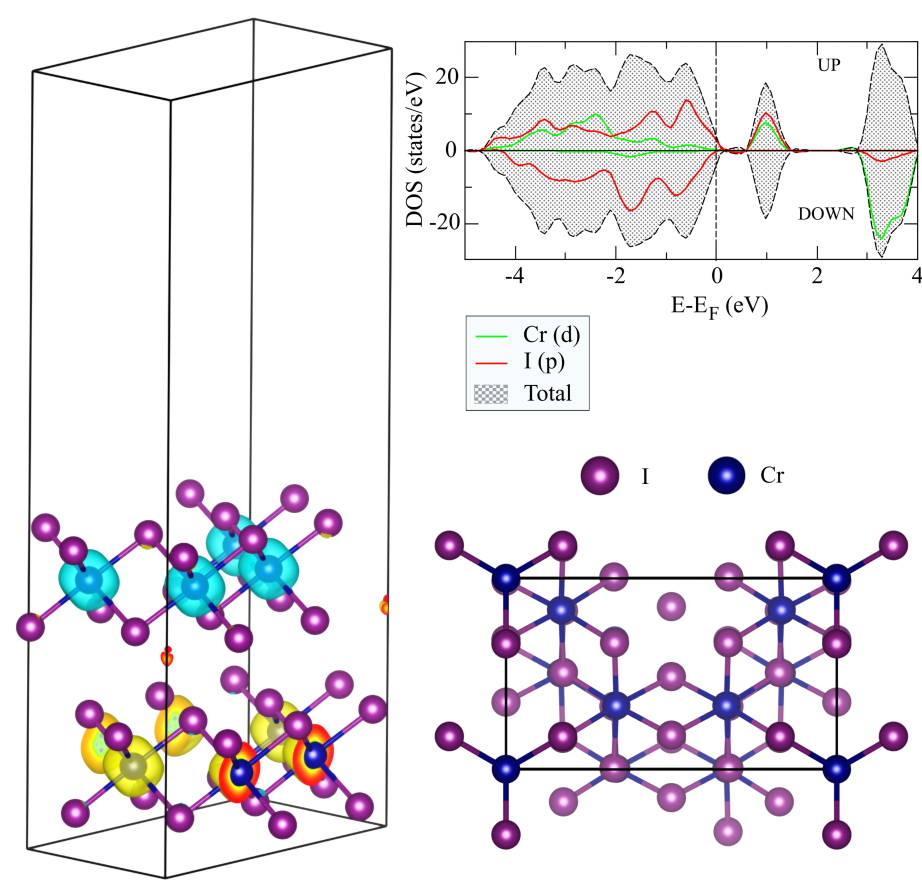

Figure 3. Orthorhombic unit cell used for simulating two $\mathrm{CrI}_{3}$ layers coupled antiferromganetically and isosurface of the magnetization density (light green for spin-up and yellow for spin-down channels correspondingly). The top view of the unit cell. Computed total and partial density of states.

\section{Accelerating with GPU Computing Example-FM-AFM Exchanges Competition in 2D Magnets}

To further examine electronic structure of $\mathrm{CrX}_{3}$ compounds we utilized a specific bonds characteristic - the electronic localization function ELF $=\left(1+\chi_{\sigma}^{2}\right)^{-1}$ (where $\chi$ is a dimensionless localization index relativity to the uniform-density electron gas). It can portray the localization nature of electrons in the system [22]. It has been calculated for all of the $\mathrm{CrX}_{3}$ materials and obviously indicates their ionic character, where electrons have been localized around halides forming $\mathrm{Cr}-\mathrm{X}$ ionic bonds. Likewise, the valency of $\mathrm{Cr}$ atoms has been computed using the Bader analysis method [23]. The increase of $\mathrm{Cr}$ ion valency along with the covalency of $p-d$ bonds was observed by going from $I$ with the biggest ionic radius up to the $F$, where the ferromagnetic exchange gets weaker and is less preferable than the anti-ferromagnetic (see Figure 4).

Results of ELF calculations for the $\mathrm{CrX}_{3}$ materials shown on the Figure 4 obviously indicate the presence of ionic character, where electrons localized around halides forming $\mathrm{Cr}-\mathrm{X}$ bonds. However, we found that the ionicity of $\mathrm{Cr}$ atoms tends to decrease going from the I up to $\mathrm{F}$ halogen by bringing more covalency to $\mathrm{Cr}-\mathrm{X}$ bonds and widening the band gap. Looking to computed charge density maps and projected crystal orbital Hamilton populations ( $\mathrm{pCOHP}$ ), one can clearly see covalent bridge formation due to the strengthening of $\mathrm{Cr}-\mathrm{X}$ bonds. Those facts are consistent with a ligand-field theory [24], where assuming constant on-cation Hubbard repulsion $U_{3 d}$ for a bigger $t_{2 g}-e_{g}$ orbitals separation $\Delta_{t_{2 g}-e_{g}}$, one can expect the FM exchange to be less preferable against the AFM contribution $J^{A F M} \approx 0.25 \Delta_{t_{2 g}-e_{g}}^{2} / U_{3 d}$ [25]. However, this kind of picture differs from the competition mechanism between ferromagnetic superexchange mediated through the $X$ ions and direct antiferromagnetic exchanges in well-studied metal halides $\mathrm{MX}_{2}$ [26-28]. Therefore, the deeper understanding of the $p-d$ hybridization in the $2 \mathrm{D} \mathrm{Cr} X_{3}$ halides is the key point for the correct description of microscopic mechanisms where indirect super-exchange will play a major role. 


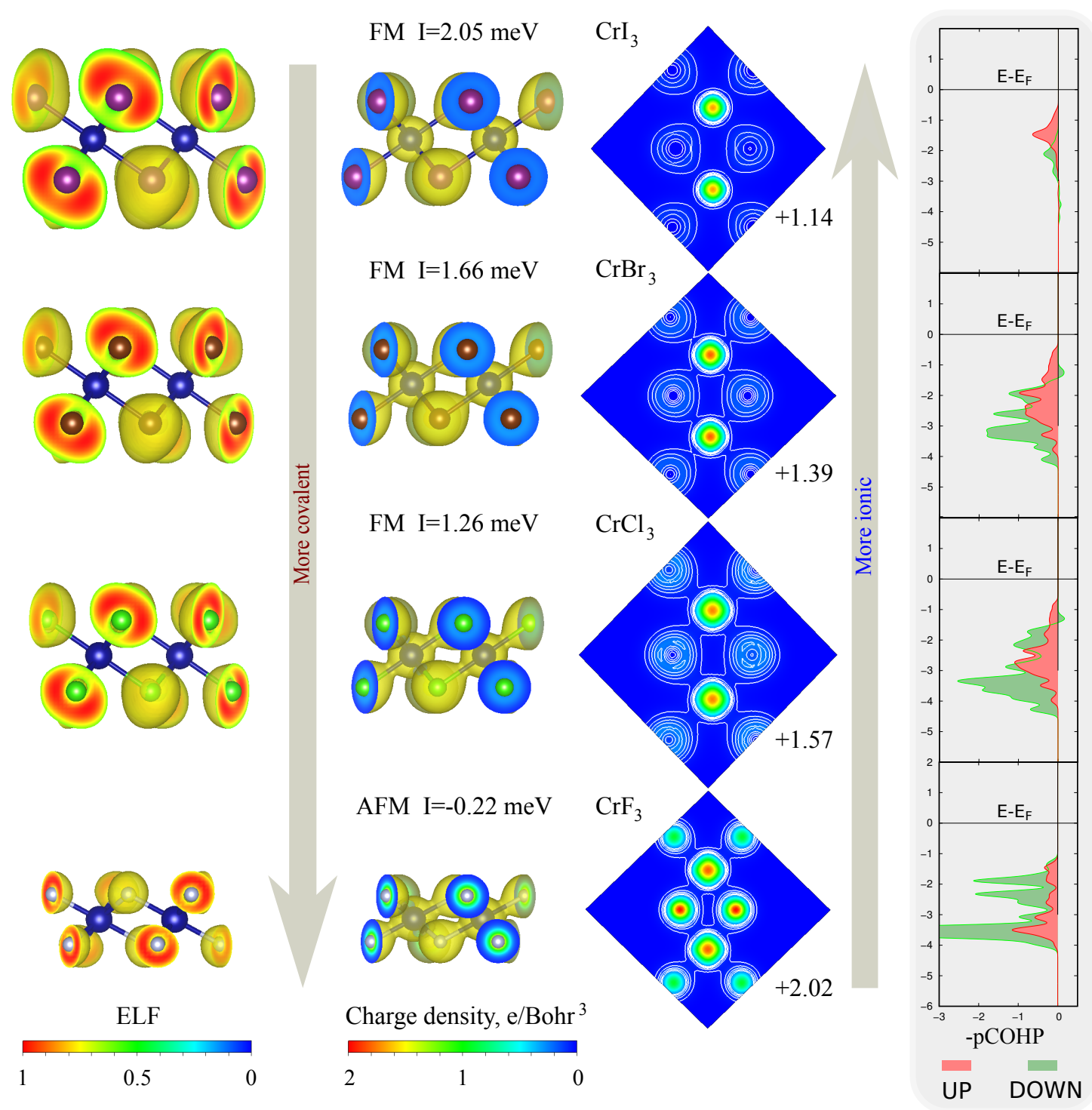

Figure 4. 3D plots are charge density maps and distributions of the electronic localization function in the unit cell of $\mathrm{CrX}_{3}$ compounds with 0.5 and $0.05 \mathrm{e} / \mathrm{Bohr}^{3}$ isosurfaces. The $2 \mathrm{D}$ cuts are charge density maps in the $(\mathrm{Cr}-\mathrm{X}-\mathrm{Cr}) \mathrm{plane}$ with $0.05 \mathrm{e} / \mathrm{Bohr}^{3}$ isolines $\left(0-0.3 \mathrm{e} / \mathrm{Bohr}^{3}\right)$ where the left and right arrows indicate the ionicity and covalency change correspondently. Numbers near the right and left arrows correspond to the Bader charges of $\mathrm{Cr}$ atoms and total magnetic exchange $J^{F M}+J^{A F M}$. pCOHP graphs for up and down spin channels placed into grey inserts indicate the strength of orbital overlaps for $\mathrm{Cr}-\mathrm{X}$ bonds where the energy axis is shown relative to the Fermi level.

For the Hubbard-like model describing magnetism of localized magnetic moments $\vec{S}_{i}$ of $d$-shells it can be shown that the second order perturbative expansion in the large Coulomb interaction limit $U_{d d}>>t_{e f f}$ yields the standard Heisenberg picture with a well know anti-ferromagnetic exchange value $J^{A F M}=-2 t_{e f f}^{2} / U_{d d}$. However, this kind of simplified picture is not suitable for a correct description of magnetic transition metal (TM) compounds where the metal atom-metal atom magnetic exchange is mediated by ligands. Therefore, even for a simple model of the metallic atoms dimer, the effect of charge transfer should be considered [29] where the total exchange $J \approx 2 l^{\prime}-4\left(t_{e f f}+l^{\prime}\right)^{2} / U_{d d}$ possesses a competitive character between FM and AFM exchanges due to the two-electron ionic integral $l^{\prime}$. Moreover, in the recent study [30], the importance of ligand-field phenomena and charge-transfer transitions for the optoelectronic properties of $1 \mathrm{~L} \mathrm{CrI}_{3}$ demonstrated. Therefore, the bigger increase of energetic cost for the charge transfer $l^{\prime}$ in comparison to the $t_{e f f}$ explains the reduction of FM exchange contribution in the $\mathrm{MX}_{3}$ halides: for the large 
halide ions, it is easy to donate electrons and form more covalent bonds. So by moving $\mathrm{X}$ in the 17th column of the periodic table from the Br with a higher ionicity up to the $\mathrm{F}$ with lesser ionicity, the ferromagnetic impact become weaker in the comparison to antiferromagnetic one. In the case of $\mathrm{CrX}_{3}$, it leads to the situation where the antiferromagnetic order become more preferable for the $\mathrm{CrF}_{3}$, in contrast to other ferromagnetic compounds of that family. On the macroscopic level, those processes can be reformulated as follows: by the band gap increase the $p-e_{g}$ coupling gets smaller since it is harder for electrons to move from $p$ to the $e_{g}$-orbitals. Hence, the $t_{2 g}-p-t_{2 g}{ }^{\prime}$ electron path corresponding to the AFM exchange become more preferable, while transfer along $t_{2 g}-p-e_{g}^{\prime}$ and $e_{g}-p-e_{g}^{\prime}$ paths are impeded, which correspond to the FM exchange between $d$ elements.

However, that simplified inclusion of charge-transfer effects into the physical model is not good enough for a proper quantitative description of magnetic phenomena in bulk TM materials, where the interplay between AFM and FM contributions is important. In a more sophisticated manner, these ratios for the $\mathrm{M}^{+3}$ sublattice in the first nearest-neighbour $\langle i, j\rangle$ approximation can be described as [31]:

$$
\begin{array}{r}
\mathcal{H}=-\sum_{\langle i, j\rangle} J^{F M}(1-\mathcal{F})\left(\vec{S}_{i} \cdot \vec{S}_{j}\right) ; \\
\mathcal{F}=\left|\frac{J^{A F M}}{J^{F M}}\right|=\frac{t_{t_{2 g}-t_{2 g^{\prime}}}^{2}}{t_{t_{2 g}-e_{2 g^{\prime}}}^{2}} \cdot\left(\frac{1+\tilde{\Delta}^{2}}{\tilde{J}_{H}}-1-\tilde{\Delta}\right),
\end{array}
$$

where quantities with a tilde-the bandgap $\tilde{\Delta}=\Delta / U$ and Hund's coupling $\tilde{J}_{H}=J_{H} / U$, are normalized to the Hubbard repulsion $U$.

By keeping a metallic ion $\mathrm{M}$ unchanged in $\mathrm{MX}_{3}$ compounds $U$ and $J_{H}$, they can be assumed as slow-changing quantities due to their local character depending on $d$-orbitals. Hence, by substituting halide atoms $X$, one can control AFM/FM contributions to the total exchange due to $t_{t_{2 g}-t_{2 g}{ }^{\prime}}, t_{t_{2 g}-e_{2 g}{ }^{\prime}}$ and $\Delta$ parameters change. Reaching the situation where $\mathcal{F} \approx 1$ it is also important to consider the effects of antisymmetric exchange interactions beyond the first coordination sphere along with the higher-order $t^{2} / U$ terms like a bi-quadratic (BQ) $B \cdot\left(\vec{S}_{i} \cdot \vec{S}_{j}\right)^{2}$ and 4-spins interactions [32]. Since for 2D magnets, BQ exchange $B \approx-2\left(t_{t_{2 g}-t_{2 g}{ }^{\prime}}^{4}+t_{t_{2 g}-e_{2 g^{\prime}}}^{4}-4 t_{t_{2 g}-t_{2 g^{\prime}}}^{2} \cdot t_{t_{2 g}-e_{2 g^{\prime}}}^{2}\right) /\left(U+J_{H}\right)^{3}$ [33] can be a comparable quantity with a bilinear (BL) exchage [34] the proper account of such effects can lead to the easy-magnetization direction switches and is, thereby, crucial for correct magnetic states descriptions $[35,36]$. The noticeable impact of the bi-quadratic exchange onto the optical absorption spectra have been observed even for a bulk ferromagnetic system, like ruby, where $\mathrm{Cr}^{+3}$ ions incorporated into octahedral positions [37].

In order to demonstrate the importance of higher-order exchanges, we performed GPU-enhanced non-collinear calculations for rotation spins in the $\mathrm{RuCl}_{3}$ compound. To extract high-order exchange we used techniques proposed by Kartsev et al. [38]. It is clearly seen from Figure 5 that the higher-order effects are in evidence, and the inclusion of BQ exchange to the model leads to a more accurate description of spin subsystem behaviour. This can be explained as an admixture effect of two pseudo-spin states $\mathcal{J}_{\text {eff }}=1 / 2$ and $\mathcal{J}_{\text {eff }}=3 / 2$ [39]. While for $S>\frac{1}{2}$ the BQ term $\left(S_{i} \cdot S_{j}\right)^{2}$ can not be reduced into a form of the usual bi-linear Heisenberg exchange $\left(S_{i} \cdot S_{j}\right)$ and, hence, is nonnegligible. The $E^{\text {tot }}(\theta)$ 30 points data have been obtained almost three times faster using GPU-aided code in comparison to code employing CPU only. 




Figure 5. The presence of the higher-order exchange can be capture by performing constrained non-collinear DFT calculations where magnetic moments rotate inplane around the principal axes. Calculated total energy $E^{\text {tot }}$ as a function of $\theta$ (dots) and different fits using the bilinear Heisenberg and the biquadratic Heisenberg model. The insets show the definition of the spins rotating by angle $\theta$ relative to the vertical axis in a unit-cell plane of $\mathrm{RuCl}_{3}$ and spin configurations at $\theta=45^{\circ}$ and $135^{\circ}$.

\section{Conclusions}

In summary, the results observed indicate the efficiency of hybrid computer clusters for collinear and noncollinear first-principle calculations where we employed the IBM ESSL mathematical library with automatic partial offloading of calculations into co-processors. This approach allowed us to increase the computational speed of the application based on the BLAS library, which originally was not designed for use on graphical processors. The proposed setup of a computing environment has been effectively applied for computing the magnetic and electronic characteristics of $2 \mathrm{D}$ magnets demonstrating the peculiar ferro- and antiferromagnetic exchange interplay and related high-order effects. Therefore, the proposed methodology will speed up computational schemes for low-dimensional materials design using the premier GPU-accelerated platforms. Accordingly, it paves the way to improve the performance of the accelerated materials design based on highthroughput computational schemes.

Author Contributions: Conceptualization, A.K., S.M. and A.C.; methodology, A.K. and S.M.; software, A.K. and S.M.; validation, A.K. and S.M.; formal analysis, A.K. and S.M.; investigation, A.K. and S.M.; resources, A.C.; data curation, A.K. and S.M.; writing-original draft preparation, A.K.; writing-review and editing, A.K. and A.C.; visualization, A.K. All authors have read and agreed to the published version of the manuscript.

Funding: The work was done with partial support by the Russian Foundation for Basic Research, project № 18-29-03196. The studies were carried out using the resources of the Center for Shared Use of Scientific Equipment "Center for Processing and Storage of Scientific Data of the Far Eastern Branch of the Russian Academy of Sciences" [40], funded by the Russian Federation represented by the Ministry of Science and Higher Education of the Russian Federation under project № 075-15-2021-663. The part of this work devoted to calculations of the non-collinear exchange-correlation interactions was supported by the Ministry of Science and Higher Education of the Russian Federation (project № 0818-2020-0005).

Institutional Review Board Statement: Not applicable.

Informed Consent Statement: Not applicable.

Data Availability Statement: Not applicable. 
Acknowledgments: The authors would like to thank Sorokin Aleksei Anatolyevich for a fruitful discussion and clear recommendations.

Conflicts of Interest: The authors declare no conflict of interest.

\section{References}

1. Huang, B.; Clark, G.; Navarro-Moratalla, E.; Klein, D.R.; Cheng, R.; Seyler, K.L.; Zhong, D.; Schmidgall, E.; McGuire, M.A.; Cobden, D.H.; et al. Layer-dependent ferromagnetism in a van der Waals crystal down to the monolayer limit. Nature 2017, 546, 270. [CrossRef]

2. Mounet, N.; Gibertini, M.; Schwaller, P.; Campi, D.; Merkys, A.; Marrazzo, A.; Sohier, T.; Castelli, I.E.; Cepellotti, A.; Pizzi, G.; et al. Two-dimensional materials from high-throughput computational exfoliation of experimentally known compounds. Nat. Nanotechnol. 2018, 13, 246-252. [CrossRef] [PubMed]

3. Haastrup, S.; Strange, M.; Pandey, M.; Deilmann, T.; Schmidt, P.S.; Hinsche, N.F.; Gjerding, M.N.; Torelli, D.; Larsen, P.M.; Riis-Jensen, A.C.; et al. The Computational 2D Materials Database: High-throughput modeling and discovery of atomically thin crystals. 2D Mater. 2018, 5, 042002. [CrossRef]

4. Choudhary, K.; Kalish, I.; Beams, R.; Tavazza, F. High-throughput Identification and Characterization of Two-dimensional Materials using Density functional theory. Sci. Rep. 2017, 7, 5179. [CrossRef]

5. Moaied, M.; Hong, J. Size-Dependent Critical Temperature and Anomalous Optical Dispersion in Ferromagnetic CrI3 Nanotubes. Nanomaterials 2019, 9, 153. [CrossRef]

6. Mejia-Parra, D.; Montoya-Zapata, D.; Arbelaiz, A.; Moreno, A.; Posada, J.; Ruiz-Salguero, O. Fast Analytic Simulation for Multi-Laser Heating of Sheet Metal in GPU. Materials 2018, 11, 2078. [CrossRef]

7. Wang, Y.; Zhao, Y.; Li, W.; Jiang, J.; Ji, X.; Zomaya, A.Y. Using a GPU to Accelerate a Longwave Radiative Transfer Model with Efficient CUDA-Based Methods. Appl. Sci. 2019, 9, 4039. [CrossRef]

8. Chandrasekaran, A.; Kamal, D.; Batra, R.; Kim, C.; Chen, L.; Ramprasad, R. Solving the electronic structure problem with machine learning. Npj Comput. Mater. 2019, 5, 1-7. [CrossRef]

9. Sorokin, A.; Malkovsky, S.; Tsoy, G.; Zatsarinnyy, A.; Volovich, K. Comparative Performance Evaluation of Modern Heterogeneous High-Performance Computing Systems CPUs. Electronics 2020, 9, 1035. [CrossRef]

10. Spiga, F.; Girotto, I. phiGEMM: A CPU-GPU Library for Porting Quantum ESPRESSO on Hybrid Systems. In Proceedings of the 2012 20th Euromicro International Conference on Parallel, Distributed and Network-Based Processing, Munich, Germany, 15-17 February 2021; IEEE Computer Society: Washington, DC, USA, 2012; pp. 368-375. [CrossRef]

11. Giannozzi, P.; Baroni, S.; Bonini, N.; Calandra, M.; Car, R.; Cavazzoni, C.; Ceresoli, D.; Chiarotti, G.L.; Cococcioni, M.; Dabo, I.; et al. QUANTUM ESPRESSO: A modular and open-source software project for quantum simulations of materials. J. Phys. Condens. Matter 2009, 21, 395502. [CrossRef]

12. Perdew, J.P.; Burke, K.; Ernzerhof, M. Generalized Gradient Approximation Made Simple. Phys. Rev. Lett. 1996, 77, 3865-3868. [CrossRef]

13. Monkhorst, H.J.; Pack, J.D. Special points for Brillouin-zone integrations. Phys. Rev. B 1976, 13, 5188-5192. [CrossRef]

14. Marzari, N.; Vanderbilt, D.; De Vita, A.; Payne, M.C. Thermal Contraction and Disordering of the Al(110) Surface. Phys. Rev. Lett. 1999, 82, 3296-3299. [CrossRef]

15. Maintz, S.; Deringer, V.L.; Tchougréeff, A.L.; Dronskowski, R. LOBSTER: A tool to extract chemical bonding from plane-wave based DFT. J. Comput. Chem. 2016, 37, 1030-1035. [CrossRef]

16. Kartsev, A.; Feya, O.D.; Bondarenko, N.; Kvashnin, A.G. Stability and magnetism of fen high-pressure phases. Phys. Chem. Chem. Phys. 2019, 21, 5262-5273. [CrossRef]

17. Wang, Z.; Qu, S.; Xiang, H.; He, Z.; Shen, J. Ferromagnetic Half-Metal Cyanamides Cr (NCN) 2 Predicted from First Principles Investigation. Materials 2020, 13, 1805. [CrossRef]

18. Li, P.; Zhao, F.; Xiao, H.; Zhang, H.; Gong, H.; Zhang, S.; Liu, Z.; Zu, X. First-Principles Study of Thermo-Physical Properties of Pu-Containing Gd2Zr2O7. Nanomaterials 2019, 9, 196. [CrossRef]

19. Dudarev, S.L.; Botton, G.A.; Savrasov, S.Y.; Humphreys, C.J.; Sutton, A.P. Electron-energy-loss spectra and the structural stability of nickel oxide: An LSDA+U study. Phys. Rev. B 1998, 57, 1505-1509. [CrossRef]

20. Liu, J.; Sun, Q.; Kawazoe, Y.; Jena, P. Exfoliating biocompatible ferromagnetic Cr-trihalide monolayers. Phys. Chem. Chem. Phys. 2016, 18, 8777-8784. [CrossRef]

21. Yang, H.C.; Gong, B.C.; Liu, K.; Lu, Z.Y. Quasi-degenerate magnetic states in $\alpha-\mathrm{RuCl}_{3}$. J. Phys. Condens. Matter 2018, $31,025803$. [CrossRef]

22. Becke, A.D.; Edgecombe, K.E. A simple measure of electron localization in atomic and molecular systems. J. Chem. Phys. 1990, 92, 5397-5403. [CrossRef]

23. Tang, W.; Sanville, E.; Henkelman, G. A grid-based Bader analysis algorithm without lattice bias. J. Phys. Condens. Matter 2009, 21, 084204. [CrossRef]

24. Griffith, J.; Orgel, L. Ligand-field theory. Q. Rev. Chem. Soc. 1957, 11, 381-393. [CrossRef]

25. Anderson, P.W. New approach to the theory of superexchange interactions. Phys. Rev. 1959, 115, 2. [CrossRef]

26. Wang, H.; Fan, F.; Zhu, S.; Wu, H. Doping enhanced ferromagnetism and induced half-metallicity in CrI3 monolayer. EPL (Europhys. Lett.) 2016, 114, 47001. [CrossRef] 
27. Zhang, W.B.; Qu, Q.; Zhu, P.; Lam, C.H. Robust intrinsic ferromagnetism and half semiconductivity in stable two-dimensional single-layer chromium trihalides. J. Mater. Chem. C 2015, 3, 12457-12468. [CrossRef]

28. Kulish, V.V.; Huang, W. Single-layer metal halides $\mathrm{MX} 2(\mathrm{X}=\mathrm{Cl}, \mathrm{Br}, \mathrm{I})$ : Stability and tunable magnetism from first principles and Monte Carlo simulations. J. Mater. Chem. C 2017, 5, 8734-8741. [CrossRef]

29. Girerd, J.J.; Journaux, Y.; Kahn, O. Natural or orthogonalized magnetic orbitals: Two alternative ways to describe the exchange interaction. Chem. Phys. Lett. 1981, 82, 534-538. [CrossRef]

30. Seyler, K.L.; Zhong, D.; Klein, D.R.; Gao, S.; Zhang, X.; Huang, B.; Navarro-Moratalla, E.; Yang, L.; Cobden, D.H.; McGuire, M.A.; et al. Ligand-field helical luminescence in a 2D ferromagnetic insulator. Nat. Phys. 2018, 14, 277-281. [CrossRef]

31. Kashin, I.; Mazurenko, V.; Katsnelson, M.; Rudenko, A. Orbitally-resolved ferromagnetism of monolayer CrI3. 2D Mater. 2020, 7, 025036. [CrossRef]

32. Bruno, P. Spin-wave theory of two-dimensional ferromagnets in the presence of dipolar interactions and magnetocrystalline anisotropy. Phys. Rev. B 1991, 43, 6015. [CrossRef] [PubMed]

33. Hoffmann, M.; Blügel, S. Systematic derivation of realistic spin models for beyond-Heisenberg solids. Phys. Rev. B 2020, 101, 024418. [CrossRef]

34. Spišák, D.; Hafner, J. Theory of bilinear and biquadratic effect ofexchange interactions in iron: Bulk and surface. J. Magn. Magn. Mater. 1997, 168, 257-268. [CrossRef]

35. Meyer, S.; Dupé, B.; Ferriani, P.; Heinze, S. Dzyaloshinskii-Moriya interaction at an antiferromagnetic interface: First-principles study of Fe/Ir bilayers on Rh (001). Phys. Rev. B 2017, 96, 094408. [CrossRef]

36. Deák, A.; Szunyogh, L.; Ujfalussy, B. Thickness-dependent magnetic structure of ultrathin Fe/Ir (001) films: From spin-spiral states toward ferromagnetic order. Phys. Rev. B 2011, 84, 224413. [CrossRef]

37. Van der Ziel, J. Spectrum of first-nearest-neighbor Cr 3+ pairs in ruby. Phys. Rev. B 1974, 9, 2846. [CrossRef]

38. Kartsev, A.; Augustin, M.; Evans, R.F.; Novoselov, K.S.; Santos, E.J. Biquadratic exchange interactions in two-dimensional magnets. Npj Comput. Mater. 2020, 6, 1-11. [CrossRef]

39. Takagi, H.; Takayama, T.; Jackeli, G.; Khaliullin, G.; Nagler, S.E. Concept and realization of Kitaev quantum spin liquids. Nat. Rev. Phys. 2019, 1, 264-280. [CrossRef]

40. Sorokin, A.A.; Makogonov, S.V.; Korolev, S.P. The Information Infrastructure for Collective Scientific Work in the Far East of Russia. Sci. Tech. Inf. Process. 2017, 44, 302-304. [CrossRef] 